\title{
Selected Extensions on Eneström-kakeya Theorem
}

\section{R. K. Pavan Kumar. Pannala ${ }^{1}$}

${ }^{1}$ Department of Mathematics, ChandigrahUniversity, Gharuan-Mohali, Punjab -140413, India. kamesh9.iit@gmail.com

Article History: Received: 11 January 2021; Accepted: 27 February 2021; Published online: 5 April 2021

\begin{abstract}
The theorem of Eneström-Kakeya is important within the hypothesis of dissemination of zeros ofpolynomials. In the literature, it can be found so many extensions on Eneström-Kakeya theorem by giving various relations between the coefficients of polynomial like increasing, decreasing, irregular order etc.This paper mainly deals with some extensions on the Abdul Aziz and B AZargar theorembygiving some relaxations to the hypothesis that the coefficients are real, positive and alternative coefficients must be in increasing order.

Key words:Eneström-Kakeya theorem, location of zeros of polynomials, bounds for zeros, coefficients of polynomials, irregular coefficients of polynomials.
\end{abstract}

\section{Introduction and statement of results}

Eneström-kakeya Theorem [7]: Given the real polynomial $f(z)=\sum_{k=0}^{n} a_{k} z^{k}$.

If $a_{0} \geq a_{1} \geq a_{2} \geq \cdots \geq a_{n-1} \geq a_{n}>0$ then $f(z) \neq 0$ for $|z|<1$.

The literature includes extensions, generalizations and refinements of Eneström-Kakeya theorem ([1-6]).

Theorem-A: If $p(z)=\sum_{k=0}^{n} a_{k} z^{k}$ is a polynomialwith $a_{n} \neq 0$, such that

$\left.\begin{array}{l}a_{n} \geq a_{n-2} \geq \cdots \geq a_{1} \text { or } a_{0}>0 \\ a_{n-1} \geq a_{n-3} \geq \cdots \geq a_{0} \text { or } a_{1}>0\end{array}\right\}$ (according as $n$ is odd or even) then all the zeros of $p(z)$ lie in the $\operatorname{disc}\left|z+\frac{a_{n-1}}{a_{n}}\right| \leq 1+\frac{a_{n-1}}{a_{n}}$.

Theorem-A is given by Abdul Aziz and B.A.Zargar[1].

The hypothesis of the theorem-A is relaxed and obtained several extensions which are enumerated as follows.

\section{Main results}

Theorem-1: If $p(z)=\sum_{k=0}^{n} a_{k} z^{k}$ is a polynomial of degree $n$ with complex coefficients such that $\left.\begin{array}{c}\left|a_{n}\right| \geq\left|a_{n-2}\right| \geq \cdots \geq\left|a_{1}\right| \text { or }\left|a_{0}\right| \\ \left|a_{n-1}\right| \geq\left|a_{n-3}\right| \geq \cdots \geq\left|a_{0}\right| \text { or }\left|a_{1}\right|\end{array}\right\}$ (according as $n$ is odd or even) and $\mid$ arg. $a_{k}-\beta \mid \leq \alpha \leq \frac{\pi}{2}$ for some real $\beta$, for $k=0(1) n$ then the bound to the location of zeros of $p(z)$ is $\left|z+\frac{a_{n-1}}{a_{n}}\right| \leq$ $\frac{\left[\left\{(\cos \alpha+\sin \alpha)\left(\left|a_{n}\right|+\left|a_{n-1}\right|\right)-\left\{(\cos \alpha+\sin \alpha-1)\left(\left|a_{1}\right|+\left|a_{0}\right|\right)\right\}+2 \sin \alpha \sum_{k=0}^{n-2}\left|a_{k}\right|\right\}\right]}{\left|a_{n}\right|}$

Following extensions can be obtained with an assumption that the real parts of the coefficients are nonnegative and satisfy the hypothesis of the theorem-A.

Theorem-2:If $p(z)=\sum_{k=0}^{n} a_{k} z^{k}$ with $a_{n} \neq 0$ such that $\left.\begin{array}{c}\alpha_{n} \geq \alpha_{n-2} \geq \cdots \geq \alpha_{1} \text { or } \alpha_{0} \geq 0 \\ \alpha_{n-1} \geq \alpha_{n-3} \geq \cdots \geq \alpha_{0} \text { or } \alpha_{1} \geq 0\end{array}\right\}$ (according as $n$ is odd or even) and $\alpha_{n}>0$ where $a_{j}=\alpha_{j}+i \beta_{j}, j=$ $0(1) n$ then a bound for zeros of $p(z)$ is $|z| \leq 1+\frac{2 \alpha_{n-1}}{\alpha_{n}}+\frac{2}{\alpha_{n}} \sum_{k=0}^{n}\left|\beta_{k}\right|$.

Theorem-3:If $p(z)=\sum_{k=0}^{n} a_{k} z^{k}$ with $a_{n} \neq 0$ such that $\left.\begin{array}{c}\alpha_{n} \geq \alpha_{n-2} \geq \cdots \geq \alpha_{1} \text { or } \alpha_{0} \geq 0 \\ \alpha_{n-1} \geq \alpha_{n-3} \geq \cdots \geq \alpha_{0} \text { or } \alpha_{1} \geq 0\end{array}\right\}$ (according as $n$ is odd or even) and $\alpha_{n}>0$ where $a_{j}=\alpha_{j}+i \beta_{j}, j=$ $0(1) n$ then a sharp bound for the zeros of $p(z)$ is $R^{*} \leq|z| \leq R$

Where 


$$
R=1+\frac{2 \alpha_{n-1}}{\alpha_{n}}+\frac{2}{\alpha_{n}} \sum_{k=0}^{n}\left|\beta_{k}\right| \text { and } R^{*}=\frac{\left|a_{0}\right|}{R^{n}\left\{2 \alpha_{n} R+(R-1)\left|\beta_{n}\right|-\left(\alpha_{0}+\left|\beta_{0}\right|\right)\right\}} .
$$

One can observe that the theorem-3 is animprovement of the theorem-2.

An extension can be obtained by including the increasing sequences between imaginary coefficients and further drop the restriction that the coefficients are non-negativein the hypothesis of the theorem-3.

Theorem-4:If $p(z)=\sum_{k=0}^{n} a_{k} z^{k}$ with $a_{n} \neq 0$ such that $\alpha_{n} \geq \alpha_{n-2} \geq \cdots \geq \alpha_{1}$ or $\alpha_{0}$ $\alpha_{n-1} \geq \alpha_{n-3} \geq \cdots \geq \alpha_{0}$ or $\alpha_{1}$ $\beta_{n} \geq \beta_{n-2} \geq \cdots \geq \beta_{1}$ or $\beta_{0}$ $\beta_{n-1} \geq \beta_{n-3} \geq \cdots \geq \beta_{0}$ or $\left.\beta_{1}\right)$ (according as $n$ is odd or even) and $\alpha_{n}>0$ where

$a_{j}=\alpha_{j}+i \beta_{j}, j=0(1) n$ then all the zeros of $p(z)$ lie in the annular ring $R_{2} \leq|z| \leq R_{1}$

where

and

$$
\begin{gathered}
R_{1}=\frac{\left|a_{n-1}\right|}{2}\left(\frac{1}{\alpha_{n}}-\frac{1}{M}\right)+\left\{\frac{\left|a_{n-1}\right|^{2}}{4}\left(\frac{1}{\alpha_{n}}-\frac{1}{M}\right)^{2}+\frac{M}{\alpha_{n}}\right\}^{\frac{1}{2}} \\
R_{2}=\frac{-R_{1}^{2}\left|a_{1}\right|\left(M_{1}-\left|a_{0}\right|\right)+\left\{R_{1}^{4}\left|a_{1}\right|^{2}\left(M_{1}-\left|a_{0}\right|\right)^{2}+4\left|a_{0}\right| R_{1}^{2} M_{1}^{3}\right\}^{\frac{1}{2}}}{2 M_{1}^{2}}
\end{gathered}
$$

$$
\begin{gathered}
M \equiv \alpha_{n}+\alpha_{n-1}+\beta_{n}+\beta_{n-1}+\left(\left|\alpha_{0}\right|-\alpha_{0}\right)+\left(\left|\alpha_{1}\right|-\alpha_{1}\right)+\left(\left|\beta_{0}\right|-\beta_{0}\right)+\left(\left|\beta_{1}\right|-\beta_{1}\right)+\left|\alpha_{n-1}\right|+\left|\beta_{n-1}\right| \\
M_{1}=R_{1}^{n+1}\left[\left(\left|\alpha_{n}\right|+\left|\beta_{n}\right|\right) R_{1}+M-\left|\alpha_{0}\right|-\left|\beta_{0}\right|\right]
\end{gathered}
$$

An extension can be obtained by including both increasing and decreasing sequences between alternative coefficients in the hypothesis of the theorem-4.

Theorem-5:If $p(z)=\sum_{k=0}^{n} a_{k} z^{k}$ with $a_{n} \neq 0$ such that $\alpha_{n} \geq \alpha_{n-2} \geq \cdots \geq \alpha_{1}$ or $\left.\alpha_{0}\right)$ $\alpha_{n-1} \leq \alpha_{n-3} \leq \cdots \leq \alpha_{0}$ or $\alpha_{1}$ $\beta_{n} \geq \beta_{n-2} \geq \cdots \geq \beta_{1}$ or $\left.\beta_{0}\right\}$ (according as $n$ is odd or even) and $\alpha_{n}>0$ where $\beta_{n-1} \leq \beta_{n-3} \leq \cdots \leq \beta_{0}$ or $\left.\beta_{1}\right)$

$a_{j}=\alpha_{j}+i \beta_{j}, j=0(1) n$ then all the zeros of $p(z)$ lie in the disc $|z| \leq \frac{M_{2}}{\alpha_{n}}$ where

$$
M_{2}=\left\{\begin{array}{r}
\alpha_{n}+\left(\left|\alpha_{0}\right|+\left|\beta_{0}\right|+\alpha_{0}+\beta_{0}\right)+\left(\left|\alpha_{1}\right|+\left|\beta_{1}\right|-\alpha_{1}\right)+\left(\left|\alpha_{n-1}\right|+\left|\beta_{n-1}\right|-\alpha_{n-1}-\beta_{n-1}\right)+\left(\beta_{n}-\beta_{1}\right) \\
\text { or } \\
\alpha_{n}+\left(\left|\alpha_{0}\right|+\left|\beta_{0}\right|-\alpha_{0}\right)+\left(\left|\alpha_{1}\right|+\left|\beta_{1}\right|+\alpha_{1}+\beta_{1}\right)+\left(\left|\alpha_{n-1}\right|+\left|\beta_{n-1}\right|-\alpha_{n-1}-\beta_{n-1}\right)+\left(\beta_{n}-\beta_{0}\right) \\
\text { accordning as n is odd or even }
\end{array}\right.
$$

\section{Lemmas} [3].

For proving the main results, the following lemmas have used. Lemma 1 owes itself to Govil and Rahman

Lemma 1: If $\left|\arg . a_{k}-\beta\right| \leq \alpha \leq \frac{\pi}{2}$, |arg. $a_{k-1}-\beta \mid \leq \alpha$ and $\left|a_{k}\right| \geq\left|a_{k-1}\right|$ then

$$
\left|a_{k}-a_{k-1}\right| \leq\left\{\left(\left|a_{k}\right|-\left|a_{k-1}\right|\right) \cos \alpha+\left(\left|a_{k}\right|+\left|a_{k-1}\right|\right) \sin \alpha\right\}
$$

One can observe that the extension of Schwarz's lemma is the following lemma 2.

Lemma 2: If $h(z)$ is analytic on and inside the unit circle, $|h(z)| \leq H$ on $|z|=1, f(0)=a$ where $|a|<H$ then $|h(z)| \leq H \frac{H|z|+|a|}{|a||z|+H}$ for $|z|<1$.

Lemma 3: If $h(z)$ is analytic in $|z|<r,|h(z)| \leq H$ on $|z|=r, h(0)=a$ where $|a|<H$ then $|h(z)| \leq H \frac{H|z|+|a| r}{|a||z|+H r}$ for $|z| \leq r$.

Lemma 3 can be proved from lemma 2 easily.

Govil, et al. [4] are attributed to the following lemma 4. 
Lemma 4: If $h(z)$ is analytic in $|z| \leq 1, h(0)=c$ where $|c|<1, h^{\prime}(0)=d,|h(z)| \leq 1$ on $|z|=1$ then for $|z| \leq 1,|h(z)| \leq \frac{(1-|c|)|z|^{2}+|d||z|+|c|(1-|c|)}{|c|(1-|c|)|z|^{2}+|d||z|+(1-|c|)}$.

Lemma 5: If $h(z)$ is analytic in $|z| \leq r, h(0)=0, h^{\prime}(0)=b$ and $|h(z)| \leq H$ for $|z|=r$ then for $|z| \leq r$, $|h(z)| \leq \frac{H|z|}{r^{2}} \frac{H|z|+r^{2}|b|}{H+|z||b|}$

Lemma 5 can be proved from lemma 4 easily.

\section{Main results proofs}

\section{Theorem-1 proof:}

$\operatorname{Let} g(z)=\left(1-z^{2}\right) p(z)$

$$
\begin{gathered}
=-a_{n} z^{n+2}-a_{n-1} z^{n+1}+\sum_{k=0}^{n-2}\left(a_{k+2}-a_{k}\right) z^{k+2}+a_{1} z+a_{0} \\
|g(z)| \geq|z|^{n+1}\left|a_{n} z+a_{n-1}\right|-\left|\sum_{k=0}^{n-2}\left(a_{k+2}-a_{k}\right) z^{k+2}+a_{1} z+a_{0}\right|
\end{gathered}
$$

For $|z|>1$,

$$
|g(z)| \geq|z|^{n+1}\left|a_{n} z+a_{n-1}\right|-|z|^{n}\left\{\sum_{k=0}^{n-2}\left|\left(a_{k+2}-a_{k}\right)\right|+\left|a_{1}\right|+\left|a_{0}\right|\right\}
$$

Using Lemma-1 we obtain

$|g(z)| \geq|z|^{n+1}\left|a_{n} z+a_{n-1}\right|-|z|^{n}\left[\left\{\sum_{k=0}^{n-2}\left(\left|a_{k+2}\right|-\left|a_{k}\right|\right) \cos \alpha\right\}+\left\{\sum_{k=0}^{n-2}\left(\left|a_{k+2}\right|+\left|a_{k}\right|\right) \sin \alpha\right\}+\left|a_{1}\right|+\left|a_{0}\right|\right]$ $=|z|^{n+1}\left|a_{n} z+a_{n-1}\right|$

$$
\begin{aligned}
& -|z|^{n}\left[\left\{(\cos \alpha+\sin \alpha)\left(\left|a_{n}\right|+\left|a_{n-1}\right|\right)\right\}+2 \sin \alpha \sum_{k=0}^{n-2}\left|a_{k}\right|\right. \\
& \left.-\left\{(\cos \alpha+\sin \alpha-1)\left(\left|a_{1}\right|+\left|a_{0}\right|\right)\right\}\right]
\end{aligned}
$$

$|g(z)|>0$ if

$\left|z+\left(\frac{a_{n-1}}{a_{n}}\right)\right|>\frac{\left[\left\{(\cos \alpha+\sin \alpha)\left(\left|a_{n}\right|+\left|a_{n-1}\right|\right)\right\}+2 \sin \alpha \sum_{k=0}^{n-2}\left|a_{k}\right|-\left\{(\cos \alpha+\sin \alpha-1)\left(\left|a_{1}\right|+\left|a_{0}\right|\right)\right\}\right]}{\left|a_{n}\right|} \equiv M$ (say)

Let $1 \leq M<R$

$$
M>\frac{\left|a_{n}\right|+\left|a_{n-1}\right|-\left|a_{1}\right|-\left|a_{0}\right|+\left|a_{1}\right|+\left|a_{0}\right|}{\left|a_{n}\right|}=1+\left|\frac{a_{n-1}}{a_{n}}\right| \geq 1
$$

Where $R=\left|z+\left(\frac{a_{n-1}}{a_{n}}\right)\right| \leq|z|+\left|\frac{a_{n-1}}{a_{n}}\right|$

$$
|z| \geq R-\left|\frac{a_{n-1}}{a_{n}}\right| \geq 1+R-M>1
$$

Hence $g(z)$ does not vanish for

$\left|z+\left(\frac{a_{n-1}}{a_{n}}\right)\right|>\frac{\left[\left\{(\cos \alpha+\sin \alpha)\left(\left|a_{n}\right|+\left|a_{n-1}\right|\right)\right\}+2 \sin \alpha \sum_{k=0}^{n-2}\left|a_{k}\right|-\left\{(\cos \alpha+\sin \alpha-1)\left(\left|a_{1}\right|+\left|a_{0}\right|\right)\right\}\right]}{\left|a_{n}\right|}$

Therefore, those roots of $g(z)$ for whichthe modulus is greater than one be located in

$\left|z+\left(\frac{a_{n-1}}{a_{n}}\right)\right| \leq \frac{\left[\left\{(\cos \alpha+\sin \alpha)\left(\left|a_{n}\right|+\left|a_{n-1}\right|\right)\right\}+2 \sin \alpha \sum_{k=0}^{n-2}\left|a_{k}\right|-\left\{(\cos \alpha+\sin \alpha-1)\left(\left|a_{1}\right|+\left|a_{0}\right|\right)\right\}\right]}{\left|a_{n}\right|}$

\section{Theorem-2 proof:}

$\operatorname{Let} g(z)=\left(1-z^{2}\right) p(z)$

$=-a_{n} z^{n+2}+Q(z)$ where $Q(z)=-a_{n-1} z^{n+1}+\sum_{k=0}^{n-2}\left(a_{k+2}-a_{k}\right) z^{k+2}+a_{1} z+a_{0}$

For $|z|=1$,

$$
|Q(z)| \leq\left|a_{0}\right|+\left|a_{1}\right|+\left|a_{n-1}\right|+\sum_{k=2}^{n}\left|a_{k}-a_{k-2}\right|
$$


Hence also

$$
\begin{gathered}
|Q(z)| \leq \alpha_{0}+\left|\beta_{0}\right|+\alpha_{1}+\left|\beta_{1}\right|+\alpha_{n-1}+\left|\beta_{n-1}\right|+\sum_{k=2}^{n}\left(\alpha_{k}-\alpha_{k-2}\right)+\sum_{k=2}^{n}\left(\left|\beta_{k}\right|+\left|\beta_{k-2}\right|\right) \\
=\alpha_{n}+2 \alpha_{n-1}-\left|\beta_{n}\right|+2 \sum_{k=0}^{n}\left|\beta_{k}\right| \\
\leq \alpha_{n}+2 \alpha_{n-1}+2 \sum_{k=0}^{n}\left|\beta_{k}\right|
\end{gathered}
$$

$$
\left|z^{n+1} Q\left(\frac{1}{z}\right)\right| \leq \alpha_{n}+2 \alpha_{n-1}+2 \sum_{k=0}^{n}\left|\beta_{k}\right|
$$

For $|z|=1$, by the maximum modulus principle holds inside the unit circle as well.

If $R>1$ then $\frac{1}{R} e^{-i \theta}$ be located in the unit circle for all real $\theta$, which implies

$\left|Q\left(R e^{i \theta}\right)\right| \leq\left\{\alpha_{n}+2 \alpha_{n-1}+2 \sum_{k=0}^{n}\left|\beta_{k}\right|\right\} R^{n+1}$ for every $R \geq 1$ and $\theta$ real.

Thus for $|z|=R>1$

$$
\begin{gathered}
\left|g\left(R e^{i \theta}\right)\right| \geq\left|a_{n}\right| R^{n+2}-\left|Q\left(R e^{i \theta}\right)\right| \\
\geq\left|a_{n}\right| R^{n+2}-\left\{\alpha_{n}+2 \alpha_{n-1}+2 \sum_{k=0}^{n}\left|\beta_{k}\right|\right\} R^{n+1} \\
\geq \alpha_{n} R^{n+2}-\left\{\alpha_{n}+2 \alpha_{n-1}+2 \sum_{k=0}^{n}\left|\beta_{k}\right|\right\} R^{n+1} \\
\left|g\left(R e^{i \theta}\right)\right|>0 \text { if } R>\frac{\left\{\alpha_{n}+2 \alpha_{n-1}+2 \sum_{k=0}^{n}\left|\beta_{k}\right|\right\}}{\alpha_{n}} .
\end{gathered}
$$

\section{Theorem-3 proof:}

$\operatorname{Let} g(z)=\left(1-z^{2}\right) p(z)$

$=a_{0}+f(z)$ where $f(z)=-a_{n} z^{n+2}-a_{n-1} z^{n+1}+\sum_{k=2}^{n}\left(a_{k}-a_{k-2}\right) z^{k}+a_{1} z$

Let $M(r)=\max _{|z|=r}|f(z)|$

Then $M(R) \geq\left|a_{0}\right|$ where $R=\frac{\left\{\alpha_{n}+2 \alpha_{n-1}+2 \sum_{k=0}^{n}\left|\beta_{k}\right|\right\}}{\alpha_{n}}$

Clearly, $|f(z)| \leq\left|a_{n}\right||z|^{n+2}+\left|a_{n-1}\right||z|^{\alpha_{n}}+\sum_{k=2}^{n}\left|a_{k}-a_{k-2}\right||z|^{k}+\left|a_{1}\right||z|$ and $R \geq 1$.

Hence,

$$
\begin{gathered}
M(R)=\max _{|z|=R}|f(z)| \leq\left|a_{n}\right| R^{n+2}+\left|a_{n-1}\right| R^{n+1}+\left|a_{1}\right| R+\sum_{k=2}^{n}\left|a_{k}-a_{k-2}\right| R^{k} \\
\leq\left|a_{n}\right| R^{n+2}+\left|a_{n-1}\right| R^{n+1}+\left|a_{1}\right| R+R^{n}\left\{\sum_{k=2}^{n}\left|a_{k}-a_{k-2}\right|\right\} \\
\leq\left|a_{n}\right| R^{n+2}+R^{n+1}\left\{\left|a_{n-1}\right|+\left|a_{1}\right|+\sum_{k=2}^{n}\left|a_{k}-a_{k-2}\right|\right\} \\
\leq\left(\alpha_{n}+\left|\beta_{n}\right|\right) R^{n+2}+R^{n+1}\left\{\alpha_{n}+2 \alpha_{n-1}-\alpha_{0}-\left|\beta_{0}\right|-\left|\beta_{n}\right|+2 \sum_{k=0}^{n}\left|\beta_{k}\right|\right\} \\
=R^{n+1}\left\{2 \alpha_{n} R+(R-1)\left|\beta_{n}\right|-\left(\alpha_{0}+\left|\beta_{0}\right|\right)\right\} \equiv M
\end{gathered}
$$

Since $f(0)=0$, hence for $|z| \leq R$ we have by Schwarz's lemma,

$$
|f(z)| \leq \frac{M|z|}{R}
$$

For $|z| \leq R,|g(z)| \geq\left|a_{0}\right|-|z| R^{n}\left\{2 \alpha_{n} R+(R-1)\left|\beta_{n}\right|-\left(\alpha_{0}+\left|\beta_{0}\right|\right)\right\}$

$|g(z)|>0$ if $|z|<\frac{\left|a_{0}\right|}{R^{n}\left\{2 \alpha_{n} R+(R-1)\left|\beta_{n}\right|-\left(\alpha_{0}+\left|\beta_{0}\right|\right)\right\}}$

Since $\frac{2 \alpha_{n} R+(R-1)\left|\beta_{n}\right|-\left(\alpha_{0}+\left|\beta_{0}\right|\right)}{\left|a_{0}\right|}>0$

Then $\frac{\left|a_{0}\right|}{R^{n}\left\{2 \alpha_{n} R+(R-1)\left|\beta_{n}\right|-\left(\alpha_{0}+\left|\beta_{0}\right|\right)\right\}}<R$.

\section{Theorem-4 proof:}

$\operatorname{Let} g(z)=\left(1-z^{2}\right) p(z)$

$=-a_{n} z^{n+2}+Q(z)$ where $\quad Q(z)=-a_{n-1} z^{n+1}+\sum_{k=2}^{n}\left(a_{k}-a_{k-2}\right) z^{k}+a_{1} z+a_{0}$

Let $T(z)=z^{n+1} Q\left(\frac{1}{z}\right)=-a_{n-1}+\sum_{k=2}^{n}\left(a_{k}-a_{k-2}\right) z^{n-k+1}+a_{1} z^{n}+a_{0} z^{n+1}$ 
For $|z|=1$, we have

$$
|T(z)| \leq\left|a_{0}\right|+\left|a_{1}\right|+\left|a_{n-1}\right|+\sum_{k=2}^{n}\left|a_{k}-a_{k-2}\right| \leq M
$$

where

$M \equiv \alpha_{n}+\alpha_{n-1}+\beta_{n}+\beta_{n-1}+\left(\left|\alpha_{0}\right|-\alpha_{0}\right)+\left(\left|\alpha_{1}\right|-\alpha_{1}\right)+\left(\left|\beta_{0}\right|-\beta_{0}\right)+\left(\left|\beta_{1}\right|-\beta_{1}\right)+\left|\alpha_{n-1}\right|+\left|\beta_{n-1}\right|$

By the maximum modulus principle, it holds inside the unit circle as well.

If $R>1$ then $\frac{1}{R} e^{-i \theta}$ be located in the unit circle for all real $\theta$,which implies

$\left|Q\left(R e^{i \theta}\right)\right| \leq M R^{n+1}$ for every real $R \geq 1$ and real $\theta$.

Thus for $|z|=R>1$

$\left|g\left(R e^{i \theta}\right)\right|>0$ if

$$
\left|g\left(R e^{i \theta}\right)\right| \geq\left|a_{n}\right| R^{n+2}-\left|Q\left(R e^{i \theta}\right)\right| \geq \alpha_{n} R^{n+2}-M R^{n+1}
$$

$R>\frac{M}{\alpha_{n}}$

$=1+\left\{\frac{\alpha_{n-1}+\beta_{n}+\beta_{n-1}+\left(\left|\alpha_{0}\right|-\alpha_{0}\right)+\left(\left|\alpha_{1}\right|-\alpha_{1}\right)+\left(\left|\beta_{0}\right|-\beta_{0}\right)+\left(\left|\beta_{1}\right|-\beta_{1}\right)+\left|\alpha_{n-1}\right|+\left|\beta_{n-1}\right|}{\alpha_{n}}\right\}$

Hence the concept of maximum modulus, $|T(0)|=\left|a_{n-1}\right|<M$

By lemma-2 on the function $T(z)$ we obtain for $|z| \leq 1$,

This implies that

$$
|T(z)| \leq M \frac{M|z|+\left|a_{n-1}\right|}{\left|a_{n-1}\right||z|+M}
$$

$$
\left|z^{n+1} Q\left(\frac{1}{z}\right)\right| \leq M \frac{M|z|+\left|a_{n-1}\right|}{\left|a_{n-1}\right||z|+M}
$$

If $R>1, \frac{1}{R} e^{-i \theta}$ be located in the unit circle for all real $\theta$, which implies

Thus for $|z|=R>1$

$$
\left|Q\left(R e^{i \theta}\right)\right| \leq M R^{n+1} \frac{M+\left|a_{n-1}\right| R}{\left|a_{n-1}\right|+M R}
$$

$$
\begin{aligned}
&\left|g\left(R e^{i \theta}\right)\right| \geq\left|a_{n}\right| R^{n+2}-\left|Q\left(R e^{i \theta}\right)\right| \geq \alpha_{n} R^{n+2}-M R^{n+1} \frac{M+\left|a_{n-1}\right| R}{\left|a_{n-1}\right|+M R} \frac{R^{n+1}}{M R+\left|a_{n-1}\right|}\left[M \alpha_{n} R^{2}-\left|a_{n-1}\right|\left(M-\alpha_{n}\right) R-M^{2}\right] \\
&>0 \text { if } R>\frac{\left|a_{n-1}\right|}{2}\left(\frac{1}{\alpha_{n}}-\frac{1}{M}\right)+\left\{\frac{\left|a_{n-1}\right|^{2}}{4}\left(\frac{1}{\alpha_{n}}-\frac{1}{M}\right)^{2}+\frac{M}{\alpha_{n}}\right\}^{\frac{1}{2}} \equiv R_{1}
\end{aligned}
$$

Therefore $g(z)$ have all the zeros of located in $|z| \leq R_{1}$ where $R_{1}>1$.

It means that all zeros of $p(z)$ are located in $|z| \leq R_{1}$.

Subsequently, it can be showed that no zeros of $p(z)$ are located in $|z|<R_{2}$.

Let $M\left(R_{1}\right)=\max _{|z|=R_{1}}|f(z)|$

$$
g(z)=a_{0}+f(z)=a_{0}+a_{1} z+\sum_{k=2}^{n}\left(a_{k}-a_{k-2}\right) z^{k}-a_{n-1} z^{n+1}-a_{n} z^{n+2}
$$

Since $R_{1} \geq 1, f(1)=-a_{0}$ we have $M\left(R_{1}\right) \geq\left|a_{0}\right|$

Clearly $|f(z)| \leq\left|a_{n}\right||z|^{n+2}+\sum_{k=2}^{n}\left|a_{k}-a_{k-2}\right||z|^{k}+\left|a_{1}\right||z|+\left|a_{n-1}\right||z|^{n+1}$

And hence $M\left(R_{1}\right) \leq\left|a_{n}\right| R_{1}^{n+2}+\sum_{k=2}^{n}\left|a_{k}-a_{k-2}\right| R_{1}^{k}+\left|a_{1}\right| R_{1}+\left|a_{n-1}\right| R_{1}^{n+1}$

$$
\leq\left|a_{n}\right| R_{1}^{n+2}+R_{1}^{n+1}\left\{\left|a_{1}\right|+\left|a_{n-1}\right|+\sum_{k=2}^{n}\left|a_{k}-a_{k-2}\right|\right\}
$$

$\leq R_{1}^{n+1}\left[\left(\left|\alpha_{n}\right|+\left|\beta_{n}\right|\right) R_{1}+M-\left|\alpha_{0}\right|-\left|\beta_{0}\right|\right] \equiv M_{1}$ (say)

Further because $f(0)=0, f^{\prime}(0)=a_{1}$ we have by lemma-5

$|f(z)| \leq \frac{M_{1}|z|}{R_{1}^{2}} \frac{M_{1}|z|+\left|R_{1}^{2}\right| a_{1} \mid}{M_{1}+\left|a_{1}\right||z|}$ for $|z| \leq R_{1}$

$$
\begin{gathered}
|g(z)| \geq\left|a_{0}\right|-\frac{M_{1}|z| M_{1}|z|+\left|R_{1}^{2}\right| a_{1} \mid}{R_{1}^{2}} \frac{-1}{M_{1}+\left|a_{1}\right||z|}=\frac{-1}{R_{1}^{2}\left(M_{1}+|z|\left|a_{1}\right|\right)}\left[|z|^{2} M_{1}^{2}+R_{1}^{2}\left|a_{1}\right||z|\left(M_{1}-\left|a_{0}\right| R_{1}^{2} M_{1}\right]\right. \\
|g(z)|>0 \text { if }|z|<\frac{-R_{1}^{2}\left|a_{1}\right|\left(M_{1}-\left|a_{0}\right|\right)+\left\{R_{1}^{4}\left|a_{1}\right|^{2}\left(M_{1}-\left|a_{0}\right|\right)^{2}+4\left|a_{0}\right| R_{1}^{2} M_{1}^{3}\right\}^{\frac{1}{2}}}{2 M_{1}^{2}} \equiv R_{2} \text { (say) where } R_{2} \leq R_{1} .
\end{gathered}
$$




\section{Theorem-5 proof:}

$\operatorname{Let} g(z)=\left(1-z^{2}\right) p(z)$

$=-a_{n} z^{n+2}+Q(z)$ where $Q(z)=-a_{n-1} z^{n+1}+\sum_{k=2}^{n}\left(a_{k}-a_{k-2}\right) z^{k}+a_{1} z+a_{0}$

For $|z|=1$ we have

where

$$
|Q(z)| \leq M_{2}
$$

$M_{2}=\left\{\begin{array}{c}\alpha_{n}+\left(\left|\alpha_{0}\right|+\left|\beta_{0}\right|+\alpha_{0}+\beta_{0}\right)+\left(\left|\alpha_{1}\right|+\left|\beta_{1}\right|-\alpha_{1}\right)+\left(\left|\alpha_{n-1}\right|+\left|\beta_{n-1}\right|-\alpha_{n-1}-\beta_{n-1}\right)+\left(\beta_{n}-\beta_{1}\right) \\ \alpha_{n}+\left(\left|\alpha_{0}\right|+\left|\beta_{0}\right|-\alpha_{0}\right)+\left(\left|\alpha_{1}\right|+\left|\beta_{1}\right|+\alpha_{1}+\beta_{1}\right)+\left(\left|\alpha_{n-1}\right|+\left|\beta_{n-1}\right|-\alpha_{n-1}-\beta_{n-1}\right)+\left(\beta_{n}-\beta_{0}\right) \\ \text { accordning as n is odd or even }\end{array}\right.$

Hence also for $|z|=1,\left|z^{n+1} Q\left(\frac{1}{z}\right)\right| \leq M_{2}$.

By the maximum modulus principle it holds inside the unit circle as well.

If $R>1$ then $\frac{1}{R} e^{-i \theta}$ be located inthe unit circle for all real $\theta$ and follows that $\left|Q\left(R e^{i \theta}\right)\right| \leq M_{2} R^{n+1}$ for every $R \geq 1$ and real $\theta$.

Thus for $|z|=R>1$

$\underset{M_{2}}{g}\left|\left(R e^{i \theta}\right)\right| \geq\left|a_{n}\right| R^{n+2}-\left|Q\left(R e^{i \theta}\right)\right| \geq \alpha_{n} R^{n+2}-M_{2} R^{n+1}$

$\left|g\left(R e^{i \theta}\right)\right|>0$ if $R>\frac{M_{2}}{\alpha_{n}}$ where $R>1$.

If $\alpha_{0}, \alpha_{1}, \alpha_{n-1} \geq 0$ and $\beta_{0}, \beta_{1}, \beta_{n-1} \geq 0$ in theorem-5 then

Corollary-5.1: If $p(z)=\sum_{k=0}^{n} a_{k} z^{k}$ with $a_{n} \neq 0$ such that $\alpha_{n} \geq \alpha_{n-2} \geq \cdots \geq \alpha_{1}$ or $\alpha_{0} \geq 0$

$0 \leq \alpha_{n-1} \leq \alpha_{n-3} \leq \cdots \leq \alpha_{0}$ or $\alpha_{1}$

$\beta_{n} \geq \beta_{n-2} \geq \cdots \geq \beta_{1}$ or $\beta_{0} \geq 0$

$0 \leq \beta_{n-1} \leq \beta_{n-3} \leq \cdots \leq \beta_{0}$ or $\left.\beta_{1}\right)$

(according as $n$ is odd or even) and $\alpha_{n}>0$

where $a_{j}=\alpha_{j}+i \beta_{j}, j=0(1) n$ then all the zeros of $p(z)$ lie in the disc

$$
|z| \leq\left\{\begin{array}{l}
\frac{\alpha_{n}+\beta_{n}+2\left(\alpha_{0}+\beta_{0}\right)}{\alpha_{n}} \\
o r \\
\frac{\alpha_{n}+\beta_{n}+2\left(\alpha_{1}+\beta_{1}\right)}{\alpha_{n}}
\end{array} \quad \text { (according as } n\right. \text { is odd or even) }
$$

If all the coefficients of the polynomial are real in theorem-5 then

Corollary-5.2: If $p(z)=\sum_{k=0}^{n} a_{k} z^{k}$ is a polynomial of degree $n$ such that

$\left.\begin{array}{c}a_{n} \geq a_{n-2} \geq \cdots \geq a_{1} \text { or } a_{0} \\ a_{n-1} \leq a_{n-3} \leq \cdots \leq a_{0} \text { or } a_{1}\end{array}\right\} \quad$ (according as $n$ is odd or even) and $a_{n}>0$ then all the roots of $p(z)$ lie in the disc

$$
|z| \leq\left\{\begin{array}{l}
1+\frac{\left(\left|a_{0}\right|+a_{0}\right)+\left(\left|a_{1}\right|-a_{1}\right)+\left(\left|a_{n-1}\right|-a_{n-1}\right)}{a_{n}} \\
1+\frac{\left(\left|a_{0}\right|-a_{0}\right)+\left(\left|a_{1}\right|+a_{1}\right)+\left(\left|a_{n-1}\right|-a_{n-1}\right)}{a_{n}}
\end{array} \text { (according as } n\right. \text { is odd or even) }
$$

If all the coefficients of the polynomial are real and non-negative in theorem-5 then

Corollary-5.3: If $p(z)=\sum_{k=0}^{n} a_{k} z^{k}$ with $a_{n} \neq 0$ such that

$a_{n} \geq a_{n-2} \geq \cdots \geq a_{1}$ or $\left.a_{0} \geq 0\right\}$

$0 \leq a_{n-1} \leq a_{n-3} \leq \cdots \leq a_{0}$ or $\left.a_{1}\right\}$

(according as $n$ is odd or even) and $a_{n}>0$ then all the roots of

$p(z)$ lie in the disc $|z| \leq\left\{\begin{array}{l}1+\frac{2 a_{0}}{a_{n}} \\ 1+\frac{2 a_{1}}{a_{n}}\end{array} \quad\right.$ (according as $n$ is odd or even) 


\section{References}

1. Aziz, A., \&Zargar, B. A. (1996). Some extensions of Enestrom-Kakeya theorem. GlasnikMatematicki, 31, 239-244.

2. Dewan, K. K., \&Bidkham, M. (1993). On the Eneström-Kakeya theorem. Journal of mathematical analysis and applications, 180(1), 29-36.

3. Govil, N. K., \& Rahman, Q. I. (1968). On the Eneström-Kakeya theorem. Tohoku Mathematical Journal, Second Series, 20(2), 126-136.

4. Govil, N. K., Rahman, Q. I., \&Schmeisser, G. (1979). On the derivative of a polynomial. Illinois Journal of Mathematics, 23(2), 319-329.

5. Gardner, R. B., \&Govil, N. K. (1994): On the Location of the Zeros of a Polynomial. Journal of Approximation Theory, 78(2), 286-292.

6. Gardner, R., \&Govil, N. (1997). Some generalizations of the Eneström-Kakeya theorem. Acta Mathematica Hungarica, 74(1-2), 125-134.

7. Marden, M. (1966). Geometry of Polynomials, Providence, RI. American Mathematical Society. 